\title{
Dynamic Behaviour under Moving Distributed Masses of Nonuniform Rayleigh Beam with General Boundary Conditions
}

\author{
Emem Ayankop Andi and Sunday Tunbosun Oni \\ Department of Mathematical Sciences, Federal University of Technology, Akure, Ondo State 340271, Nigeria \\ Correspondence should be addressed to Emem Ayankop Andi; ememandi@yahoo.com
}

Received 3 December 2013; Accepted 23 January 2014; Published 23 March 2014

Academic Editors: B. Sun and J. Sun

Copyright ( 2014 E. A. Andi and S. T. Oni. This is an open access article distributed under the Creative Commons Attribution License, which permits unrestricted use, distribution, and reproduction in any medium, provided the original work is properly cited.

\begin{abstract}
This paper investigates the flexural vibration of a finite nonuniform Rayleigh beam resting on an elastic foundation and under travelling distributed loads. For the solution of this problem, in the first instance, the generalized Galerkin method was used. The resulting Galerkin's equations were then simplified using the modified asymptotic method of Struble. The simplified second-order ordinary differential equation was then solved using the method of integral transformation. The closed form solution obtained was analyzed and results show that, an increase in the values of foundation moduli $K$ and rotatory inertia correction factor $R_{0}$ reduces the response amplitudes of both the clamped-clamped nonuniform Rayleigh beam and the clamped-free nonuniform Rayleigh beam. Also for the same natural frequency, the critical speed for the moving distributed mass problem is smaller than that for the moving distributed force problem. Hence resonance is reached earlier in the former. Furthermore, resonance conditions for the dynamical system are attained significantly by both $R_{0}$ and $K$ for the illustrative end conditions considered.
\end{abstract}

\section{Introduction}

This paper is sequel to an earlier one by Oni and AyankopAndi in [1] that considered the response of a simply supported nonuniform Rayleigh beam to travelling distributed loads. In particular, this paper is a generalization of the theory advanced in [1]. For more than a century, the analysis of continuous elastic system subjected to moving systems has been the subject of interest in many fields, from structural to mechanical to aerospace engineering. Various structures ranging from bridges and roads to space vehicles and submarines are constantly acted upon by moving masses and hence the problem of analyzing the dynamic response of these structures under the action of moving masses continues to motivate a variety of investigations. In most of the studies available in literature, such as the works of Sadiku and Leipholz in [2], Oni in [3], Gbadeyan and Oni in [4], Huang and Thambiratnam in [5], Lee and $\mathrm{Ng}$ in [6], Adams in [7], Chen and Li in [8], Savin in [9], Rao in [10], Shadnam et al. in [11], and Oni and Awodola in [12], the scope has been restricted to structural members having uniform crosssection whether the inertia of the moving load is considered or not and the load modelled as moving concentrated load.
In practice, cross-sections of elastic structures such as plates and beams are not usually uniform and the moving loads are commonly in distributed forms. To this end, in [13] an attempt was made on the studies concerning nonuniform structural members subjected to moving loads. In particular, he investigated the response of nonuniform beams resting on elastic foundation to several moving masses. He found that the response amplitudes of both moving force and moving mass problems decrease with increasing foundation constant and that the maximum transverse deflection of the beam is always greater than the displacement of the moving concentrated mass. For both clamped-clamped and clampedfree nonuniform Rayleigh beam under moving distributed loads, the response amplitudes of the beam not resting on elastic subgrade are greater than that of the beam supported by a subgrade. For these boundary conditions, the response amplitudes are lower than those of the simply supported boundary conditions. Other researchers who have worked on transverse motions under moving load of beams with nonuniform cross-sections include [14-17]. However, the scope of their consideration does not cover the wide range of application areas often encountered in engineering practice. 
They simplified their investigation by modelling their loads as concentrated line loads.

Emphatically speaking, in order to accurately model such physical situations in realistic manners, an accurate representation of the moving load is that it is distributed over a portion or over the entire length of the structure. In the light of this, recently, some researchers have addressed this shortcoming. Among these are Esmailzadez and Ghorashi in [18], Gbadeyan and Dada in [19], and Bogacz and Czyczula in [20], to mention a few who have tackled dynamical problems involving the response of elastic structures subjected to partially distributed moving masses. However their method of solution is either restricted to numerical development or suitable only for simply supported end conditions or both. In the context of this, this present paper considers the dynamic behaviour under moving distributed masses of nonuniform Rayleigh beams with general boundary conditions. The interest is in analytical development and as such often sheds valuable light on vital information about the physical system.

\section{The Governing Equation}

The nonuniform Rayleigh beam is a spatially onedimensional elastic system. Thus, the problem of vibration of the finite nonuniform Rayleigh beam of length $L$, on a Winkler foundation and traversed by a travelling distributed load whose load inertia is not negligible with damping neglected, is governed by the fourth-order partial differential equation given in [21] by Fryba as follows:

$$
\begin{aligned}
\frac{\partial^{2}}{\partial x^{2}}[E I(x)] \frac{\partial^{2} V(x, t)}{\partial x^{2}}+\mu(x) \frac{\partial^{2} V(x, t)}{\partial t^{2}} \\
-\mu(x) R_{0} \frac{\partial^{4} V(x, t)}{\partial x^{2} \partial t^{2}}+K V(x, t) \\
=M g H(x-c t)\left[1-\frac{1}{g} \frac{d^{2} V(x, t)}{d t^{2}}\right],
\end{aligned}
$$

where $x$ is the spatial coordinate, $t$ is the time, $V(x, t)$ is the transverse displacement, $E$ is Young's modulus, $R_{0}$ is the measure of rotatory inertia correction factor, and $K$ is the elastic foundation moduli and the beam properties such as the moment of inertia $I(x)$ and mass per unit length $\mu(x)$ of the beam are considered as varying along the length $L$ of the beam. $c$ is the velocity of the distributed mass $M$, and the time $t$ is assumed to be limited to that interval of time within which the mass $\mu$ is on the beam; that is,

$$
0 \leq c t \leq L \text {. }
$$

$g$ is the acceleration due to gravity, and $H(x-c t)$ is the Heaviside function defined as

$$
H(x-c t)= \begin{cases}0, & x<c t \\ 1, & x>c t\end{cases}
$$

with the following properties:

$$
\begin{aligned}
& \text { (i) } \frac{d}{d x}\{H(x-c t)\}=\delta(x-c t) \\
& \text { (ii) } f(x) H(x-c t)= \begin{cases}0, & x<c t \\
f(x), & x>c t,\end{cases}
\end{aligned}
$$

where $\delta(x-c t)$ represents the Dirac delta function defined as follows:

$$
\text { (i) } \delta(x-c t)= \begin{cases}0, & x \neq c t \\ \infty, & x=c t\end{cases}
$$

while

$$
\text { (ii) } \delta(x)= \begin{cases}0, & x \neq 0 \\ \infty, & x=0\end{cases}
$$

with the following properties:

(i) $\delta(-x)=\delta(x)$

(ii) $\int_{a}^{b} \delta(x-k) f(x) d x= \begin{cases}0, & k<a<b \\ f(k), & a<k<b \\ 0, & a<b<k\end{cases}$

$d^{2} / d t^{2}$ is the convective acceleration operator defined as follows:

$$
\frac{d^{2}}{d t^{2}}=\frac{\partial^{2}}{\partial t^{2}}+2 c \frac{\partial^{2}}{\partial x \partial t}+c^{2} \frac{\partial^{2}}{\partial x^{2}}
$$

when the mass $M$ moves with a constant velocity.

The boundary conditions of the above problem are assumed to be general, while the initial conditions are

$$
V(x, 0)=V_{t}(x, 0)=0 .
$$

Adopting examples in [22], $I(x)$ and $\mu(x)$ are given by Williams as

$$
\begin{aligned}
& I(x)=I_{0}\left(1+\operatorname{Sin} \frac{\pi x}{L}\right)^{3} \\
& \mu(x)=\mu_{0}\left(1+\operatorname{Sin} \frac{\pi x}{L}\right) .
\end{aligned}
$$




\section{Method of Solution}

Substituting (8), (10) into (1), after some simplifications and rearrangement, one obtains

$$
\begin{aligned}
& E I_{0}\left[\frac { \partial ^ { 4 } V ( x , t ) } { \partial x ^ { 4 } } \left\{\frac{5}{2}+\frac{15}{4} \operatorname{Sin} \frac{\pi x}{L}-\frac{1}{4} \operatorname{Sin} \frac{3 \pi x}{L}\right.\right. \\
&\left.-\frac{3}{2} \operatorname{Cos} \frac{2 \pi x}{L}\right\}+\frac{\partial^{2} V(x, t)}{\partial x^{2}} \\
&\left.\times\left\{\frac{9 \pi^{2}}{4 L^{2}} \operatorname{Sin} \frac{3 \pi x}{L}-\frac{15 \pi^{2}}{4 L^{2}} \operatorname{Sin} \frac{\pi x}{L}+\frac{6 \pi^{2}}{L^{2}} \operatorname{Cos} \frac{2 \pi x}{L}\right\}\right] \\
&+ \mu_{0}\left(1+\operatorname{Sin} \frac{\pi x}{L}\right) \frac{\partial^{2} V(x, t)}{\partial t^{2}}-\mu_{0} R_{0} \\
& \times {\left[\left(1+\operatorname{Sin} \frac{\pi x}{L}\right) \frac{\partial^{4} V(x, t)}{\partial x^{2} \partial t^{2}}+\frac{\pi}{L} \operatorname{Cos} \frac{\pi x}{L} \cdot \frac{\partial^{3} V(x, t)}{\partial x \partial t^{2}}\right] } \\
&+ K V(x, t)+M H(x-c t)\left[\frac{\partial^{2} V(x, t)}{\partial t^{2}}+2 c \frac{\partial^{2} V(x, t)}{\partial x \partial t}\right. \\
&= M g H(x-c t) .
\end{aligned}
$$

Equation (11) is the fourth-order partial differential equation with variable coefficients of the nonuniform Rayleigh beam under the action of travelling distributed loads. It is evident that exact closed form solution to this equation is impossible. To this end, an approximate analytical solution is desirable. An elegant approximation technique due to Galerkin referred to as Generalized Galerkin Method (GGM) discussed in [3] is employed to simplify and reduce (11) to a sequence of second-order ordinary differential equations called Galerkin equations. This technique requires that solution to (11) be written in the following form:

$$
V_{n}(x, t)=\sum_{m=1}^{n} W_{m}(t) U_{m}(x),
$$

where $U_{m}(x)$ is chosen such that the pertinent boundary conditions are satisfied. Equation (12) when substituted into (11) yields

$$
\begin{gathered}
\sum_{m=1}^{n}\left\{\frac { E I _ { 0 } } { \mu _ { 0 } } \left[\left(\frac{5}{2}+\frac{15}{4} \operatorname{Sin} \frac{\pi x}{L}-\frac{1}{4} \operatorname{Sin} \frac{3 \pi x}{L}-\frac{3}{2} \operatorname{Cos} \frac{2 \pi x}{L}\right)\right.\right. \\
\times U_{m}^{\prime v}(x)+\left(\frac{9 \pi^{2}}{4 L^{2}} \operatorname{Sin} \frac{3 \pi x}{L}-\frac{15 \pi^{2}}{4 L^{2}} \operatorname{Sin} \frac{\pi x}{L}\right. \\
\left.\left.+\frac{6 \pi^{2}}{L^{2}} \operatorname{Cos} \frac{2 \pi x}{L}\right) U_{m}^{\prime \prime}(x)\right] \\
\times W_{m}(t)+\left(1+\operatorname{Sin} \frac{\pi x}{L}\right) U_{m}(x) \ddot{W}_{m}(t)
\end{gathered}
$$

$$
\begin{aligned}
& -R_{0}\left[\left(1+\operatorname{Sin} \frac{\pi x}{L}\right) U_{m}^{\prime \prime}(x)+\left(\frac{\pi}{L} \operatorname{Cos} \frac{\pi x}{L}\right) U_{m}^{\prime}(x)\right] \\
& \times \ddot{W}_{m}(t)+\frac{K}{\mu_{0}} W_{m}(t) U_{m}(x)+\frac{M H(x-c t)}{\mu_{0}} \\
& \times\left[\ddot{W}_{m}(t) U_{m}(x)+2 c \dot{W}_{m}(t) U_{m}^{\prime}(x)\right. \\
& \left.\left.+c^{2} W_{m}(t) U_{m}^{\prime \prime}(x)\right]\right\}-\frac{M g}{\mu_{0}} H(x-c t)=0 .
\end{aligned}
$$

In order to determine $W_{m}(t)$, it is required that the expression on the left-hand side of (13) be orthogonal to the function $U_{k}(x)$. Hence,

$$
\begin{aligned}
\int_{0}^{L}\left(\sum _ { m = 1 } ^ { n } \left\{\frac { E I _ { 0 } } { \mu _ { 0 } } \left[\left(\frac{5}{2}+\frac{15}{4} \operatorname{Sin} \frac{\pi x}{L}-\frac{1}{4} \operatorname{Sin} \frac{3 \pi x}{L}\right.\right.\right.\right. \\
\left.\quad-\frac{3}{2} \operatorname{Cos} \frac{2 \pi x}{L}\right) U_{m}^{\prime v}(x) \\
+\left(\frac{9 \pi^{2}}{4 L^{2}} \operatorname{Sin} \frac{3 \pi x}{L}-\frac{15 \pi^{2}}{4 L^{2}} \operatorname{Sin} \frac{\pi x}{L}\right. \\
\left.\left.\quad+\frac{6 \pi^{2}}{L^{2}} \operatorname{Cos} \frac{2 \pi x}{L}\right) U_{m}^{\prime \prime}(x)\right] \\
\times\left[\left(1+W_{m}(t)+\left(1+\operatorname{Sin} \frac{\pi x}{L}\right) U_{m}^{\prime \prime}(x)+\left(\frac{\pi}{L} \operatorname{Cos} \frac{\pi x}{L}\right) U_{m}^{\prime}(x)\right]\right. \\
\times \ddot{W}_{m}(t)+\frac{K}{\mu_{0}} W_{m}(t) U_{m}(x)+\frac{M H(x-c t)}{\mu_{0}} \\
\times\left[\ddot{W}_{m}(t) U_{m}(x)+2 c \dot{W}_{m}(t) U_{m}^{\prime}(x)\right. \\
\left.\left.\left.\quad+c^{2} W_{m}(t) U_{m}^{\prime \prime}(x)\right]\right\}-\frac{M g}{\mu_{0}} H(x-c t)\right)
\end{aligned}
$$$$
\times U_{k}(x) d x=0 \text {. }
$$

A rearrangement of the above equation yields

$$
\begin{aligned}
\sum_{m=1}^{n}\{[ & \left(Q_{1}(m, k)+Q_{3}(m, k)\right) \\
& -R_{0}\left(Q_{4}(m, k)+Q_{2}(m, k)\right. \\
& \left.\left.+Q_{5}(m, k)\right)\right] \ddot{W}_{m}(t) \\
+ & {\left[P_{1}\left(T_{0}+T_{1}\right)+\frac{K}{\mu_{0}} Q_{1}(m, k)\right] W_{m}(t)+\frac{M}{\mu_{0}} } \\
\times & {\left[Q_{1}(t) \ddot{W}_{m}(t)+2 c Q_{2}(t) \dot{W}_{m}(t)\right.} \\
& \left.\left.+c^{2} Q_{3}(t) W_{m}(t)\right]\right\}-\frac{M g}{\mu_{0}} Q_{4}(t)=0,
\end{aligned}
$$


where

$$
\begin{aligned}
& T_{0}=Q_{9}(m, k)+Q_{10}(m, k)-\left(Q_{11}(m, k)+Q_{12}(m, k)\right) \\
& T_{1}=Q_{6}(m, k)+Q_{7}(m, k)-Q_{8}(m, k), \\
& P_{1}=\frac{E I_{0}}{\mu_{0}} \\
& Q_{1}(t)=\int_{0}^{L} H(x-c t) U_{m}(x) U_{k}(x) d x \\
& Q_{2}(t)=\int_{0}^{L} H(x-c t) U_{m}^{\prime}(x) U_{k}(x) d x \\
& Q_{3}(t)=\int_{0}^{L} H(x-c t) U_{m}^{\prime \prime}(x) U_{k}(x) d x \\
& Q_{4}(t)=\int_{0}^{L} H(x-c t) U_{k}(x) d x \\
& Q_{1}(m, k)=\int_{0}^{L} U_{m}(x) U_{k}(x) d x \\
& Q_{2}(m, k)=\int_{0}^{L} U_{m}^{\prime \prime}(x) U_{k}(x) d x \\
& Q_{3}(m, k)=\int_{0}^{L} \operatorname{Sin} \frac{\pi x}{L} U_{m}(x) U_{k}(x) d x \\
& Q_{4}(m, k)=\frac{\pi}{L} \int_{0}^{L} \operatorname{Cos} \frac{\pi x}{L} U_{m}^{\prime}(x) U_{k}(x) d x \\
& Q_{5}(m, k)=\int_{0}^{L} \operatorname{Sin} \frac{\pi x}{L} U_{m}^{\prime \prime}(x) U_{k}(x) d x \\
& Q_{6}(m, k)=\frac{9 \pi^{2}}{4 L^{2}} \int_{0}^{L} \operatorname{Sin} \frac{3 \pi x}{L} U_{m}^{\prime \prime}(x) U_{k}(x) d x \\
& Q_{7}(m, k)=\frac{6 \pi^{2}}{L^{2}} \int_{0}^{L} \operatorname{Cos} \frac{2 \pi x}{L} U_{m}^{\prime \prime}(x) U_{k}(x) d x \\
& Q_{8}(m, k)=\frac{15 \pi^{2}}{4 L^{2}} \int_{0}^{L} \operatorname{Sin} \frac{\pi x}{L} U_{m}^{\prime \prime}(x) U_{k}(x) d x \\
& Q_{9}(m, k)=\frac{5}{2} \int_{0}^{L} U_{m}^{\prime v}(x) U_{k}(x) d x \\
& Q_{10}(m, k)=\frac{15}{4} \int_{0}^{L} \operatorname{Sin} \frac{\pi x}{L} U_{m}^{\prime v}(x) U_{k}(x) d x \\
& Q_{11}(m, k)=\frac{1}{4} \int_{0}^{L} \operatorname{Sin} \frac{3 \pi x}{L} U_{m}^{\prime v}(x) U_{k}(x) d x \\
& Q_{12}(m, k)=\frac{3}{2} \int_{0}^{L} \operatorname{Cos} \frac{2 \pi x}{L} U_{m}^{\prime v}(x) U_{k}(x) d x .
\end{aligned}
$$

The ordinary differential equation (15) is valid for all variants of classical boundary conditions. Using the Fourier series representation of the Heaviside unit step function, namely,

$$
\begin{aligned}
& H(x-c t) \\
& \quad=\frac{1}{4}+\frac{1}{\pi} \sum_{n=0}^{\infty} \frac{\operatorname{Sin}((2 n+1) \pi(x-c t))}{2 n+1}, \quad 0<x<L
\end{aligned}
$$

in (15) and substituting (17) into (15) and simplifying yield

$$
\begin{gathered}
\sum_{m=1}^{n}\left\{\left[\left(Q_{1}(m, k)+Q_{3}(m, k)\right)-R_{0}\left(Q_{4}(m, k)+Q_{2}(m, k)\right.\right.\right. \\
\left.\left.\quad+Q_{5}(m, k)\right)\right] \ddot{W}_{m}(t) \\
+\left[P_{1}\left(T_{0}+T_{1}\right)+\frac{K}{\mu_{0}} Q_{1}(m, k)\right] W_{m}(t)+\frac{M}{\mu_{0}} \\
\times\left[\left(Q_{1 A}(m, k)+\frac{1}{\pi} \sum_{n=0}^{\infty} \frac{\operatorname{Cos}(2 n+1) \pi c t}{2 n+1} Q_{1 B}(n, m, k)\right.\right. \\
\left.-\frac{1}{\pi} \sum_{n=0}^{\infty} \frac{\operatorname{Sin}(2 n+1) \pi c t}{2 n+1} Q_{1 C}(n, m, k)\right) \ddot{W}_{m}(t) \\
+2 c\left(Q_{2 A}(m, k)\right. \\
+\frac{1}{\pi} \sum_{n=0}^{\infty} \frac{\operatorname{Cos}(2 n+1) \pi c t}{2 n+1} Q_{2 B}(n, m, k) \\
\left.-\frac{1}{\pi} \sum_{n=0}^{\infty} \frac{\operatorname{Sin}(2 n+1) \pi c t}{2 n+1} Q_{2 C}(n, m, k)\right) \dot{W}_{m}(t) \\
\left.\left.\times W_{m}(t)\right]\right\}=\frac{M g}{\mu_{0}} Q_{4}(t), \\
+c^{2}\left(Q_{3 A}(m, k)\right. \\
+\frac{1}{\pi} \sum_{n=0}^{\infty} \frac{\operatorname{Cos}(2 n+1) \pi c t}{2 n+1} Q_{3 B}(n, m, k) \\
\left.\frac{\operatorname{Sin}(2 n+1) \pi c t}{2 n+1} Q_{3 C}(n, m, k)\right)
\end{gathered}
$$

where

$$
\begin{gathered}
Q_{1 A}(m, k)=\frac{1}{4} \int_{0}^{L} U_{m}(x) U_{k}(x) d x \\
Q_{1 B}(n, m, k)=\int_{0}^{L} \operatorname{Sin}(2 n+1) \pi x U_{m}(x) U_{k}(x) d x \\
Q_{1 C}(n, m, k)=\int_{0}^{L} \operatorname{Cos}(2 n+1) \pi x U_{m}(x) U_{k}(x) d x \\
Q_{2 A}(m, k)=\frac{1}{4} \int_{0}^{L} U_{m}^{\prime}(x) U_{k}(x) d x \\
Q_{2 B}(n, m, k)=\int_{0}^{L} \operatorname{Sin}(2 n+1) \pi x U_{m}^{\prime}(x) U_{k}(x) d x \\
Q_{2 C}(n, m, k)=\int_{0}^{L} \operatorname{Cos}(2 n+1) \pi x U_{m}^{\prime}(x) U_{k}(x) d x \\
Q_{3 A}(m, k)=\frac{1}{4} \int_{0}^{L} U_{m}^{\prime \prime}(x) U_{k}(x) d x
\end{gathered}
$$




$$
\begin{aligned}
Q_{3 B}(n, m, k) & =\int_{0}^{L} \operatorname{Sin}(2 n+1) \pi x U_{m}^{\prime \prime}(x) U_{k}(x) d x \\
Q_{3 C}(n, m, k) & =\int_{0}^{L} \operatorname{Cos}(2 n+1) \pi x U_{m}^{\prime \prime}(x) U_{k}(x) d x .
\end{aligned}
$$

In order to solve this equation, an appropriate selection of function is the following beam function $U_{m}(x)$ :

$$
\begin{aligned}
U_{m}(x)= & \operatorname{Sin} \frac{\lambda_{m} x}{L}+A_{m} \operatorname{Cos} \frac{\lambda_{m} x}{L}+B_{m} \operatorname{Sinh} \frac{\lambda_{m} x}{L} \\
& +C_{m} \operatorname{Cosh} \frac{\lambda_{m} x}{L}
\end{aligned}
$$

so that

$$
\begin{aligned}
U_{k}(x)= & \operatorname{Sin} \frac{\lambda_{k} x}{L}+A_{k} \operatorname{Cos} \frac{\lambda_{k} x}{L}+B_{k} \operatorname{Sinh} \frac{\lambda_{k} x}{L} \\
& +C_{k} \operatorname{Cosh} \frac{\lambda_{k} x}{L} .
\end{aligned}
$$

The constants $A_{m}, B_{m}, C_{m}, A_{k}, B_{k}, C_{k}$ and the mode frequencies $\lambda_{m}, \lambda_{k}$ are usually determined by using the appropriate classical boundary conditions. Substituting (20) and (21) into (18), after some simplification and rearrangement, one obtains

$$
\begin{gathered}
\sum_{m=1}^{n}\left\{\Delta_{1}(m, k) \ddot{W}_{m}(t)+\Delta_{2}(m, k) W_{m}(t)+\epsilon_{a} L\right. \\
\times\left[\left(Q_{1 A}(m, k)+\frac{1}{\pi} \sum_{n=0}^{\infty} \frac{\operatorname{Cos}(2 n+1) \pi c t}{2 n+1} Q_{1 B}(n, m, k)\right.\right. \\
\left.\quad-\frac{1}{\pi} \sum_{n=0}^{\infty} \frac{\operatorname{Sin}(2 n+1) \pi c t}{2 n+1} Q_{1 C}(n, m, k)\right) \ddot{W}_{m}(t)+2 c \\
\times\left(Q_{2 A}(m, k)+\frac{1}{\pi} \sum_{n=0}^{\infty} \frac{\operatorname{Cos}(2 n+1) \pi c t}{2 n+1} Q_{2 B}(n, m, k)\right. \\
\left.\quad-\frac{1}{\pi} \sum_{n=0}^{\infty} \frac{\operatorname{Sin}(2 n+1) \pi c t}{2 n+1} Q_{2 C}(n, m, k)\right) \dot{W}_{m}(t) \\
+c^{2}\left(Q_{3 A}(m, k)\right. \\
\left.\left.\times W_{m}(t)\right]\right\} \\
\quad+\frac{1}{\pi} \sum_{n=0}^{\infty} \frac{\operatorname{Cos}(2 n+1) \pi c t}{2 n+1} Q_{3 B}(n, m, k) \\
2 n+1 \\
\quad \operatorname{Sin}(2 n+1) \pi c t \\
\left.Q_{3 C}(n, m, k)\right)
\end{gathered}
$$

$$
\begin{gathered}
=\frac{\epsilon_{a} g L^{2}}{\lambda_{k}}\left[B_{f}(\lambda, k)+\operatorname{Cos} \frac{\lambda_{k} c t}{L}-A_{k} \operatorname{Sin} \frac{\lambda_{k} c t}{L}\right. \\
\left.-B_{k} \operatorname{Cosh} \frac{\lambda_{k} c t}{L}-C_{k} \operatorname{Sinh} \frac{\lambda_{k} c t}{L}\right],
\end{gathered}
$$

where

$$
\begin{gathered}
\Delta_{1}(m, k)=Q_{1}(m, k)+Q_{3}(m, k) \\
-R_{0}\left(Q_{4}(m, k)+Q_{2}(m, k)+Q_{5}(m, k)\right) \\
\Delta_{2}(m, k)=P_{1}\left(T_{0}+T_{1}\right)+\frac{K}{\mu_{0}} Q_{1}(m, k), \\
\epsilon_{a}=\frac{M}{\mu_{0} L} \\
B_{f}(\lambda, k)=-\operatorname{Cos} \lambda_{k}+A_{k} \operatorname{Sin} \lambda_{k}+B_{k} \operatorname{Cosh} \lambda_{k}+C_{k} \operatorname{Sinh} \lambda_{k} .
\end{gathered}
$$

Equation (22) is now the fundamental equation governing the problem of flexural vibrations of a finite nonuniform Rayleigh beam under travelling distributed loads. In what follows, two special cases of (22) are discussed.

\section{Closed Form Solution}

4.1. Case I. The differential equation describing the flexural vibrations of a finite nonuniform Rayleigh beam subjected to a moving distributed force may be obtained from (22) by setting $\epsilon_{a}=0$. In this case, we obtain

$$
\begin{aligned}
\sum_{m=1}^{n}\left\{\Delta_{1}(m, k) \ddot{W}_{m}(t)+\Delta_{2}(m, k) W_{m}(t)\right\} & \\
=\frac{M g L}{\mu_{0} \lambda_{k}}[ & B_{f}(\lambda, k)+\operatorname{Cos} \frac{\lambda_{k} c t}{L}-A_{k} \operatorname{Sin} \frac{\lambda_{k} c t}{L} \\
& \left.-B_{k} \operatorname{Cosh} \frac{\lambda_{k} c t}{L}-C_{k} \operatorname{Sinh} \frac{\lambda_{k} c t}{L}\right] .
\end{aligned}
$$

Rearranging (24) and ignoring the summation sign yield

$$
\begin{aligned}
\ddot{W}_{m}(t)+\beta_{m}^{2} W_{m}(t) & \\
=P_{0}[ & B_{f}(\lambda, k)+\operatorname{Cos} \frac{\lambda_{k} c t}{L}-A_{k} \operatorname{Sin} \frac{\lambda_{k} c t}{L} \\
& \left.\quad-B_{k} \operatorname{Cosh} \frac{\lambda_{k} c t}{L}-C_{k} \operatorname{Sinh} \frac{\lambda_{k} c t}{L}\right],
\end{aligned}
$$

where

$$
\beta_{m}^{2}=\frac{\Delta_{2}(m, k)}{\Delta_{1}(m, k)}, \quad P_{0}=\frac{M g L}{\mu_{0} \lambda_{k} \Delta_{1}(m, k)} .
$$


Solving (25) in conjunction with the initial condition, the solution is given by

$$
\begin{aligned}
W_{m}(t)=P_{0}\{ & B_{f}(\lambda, k)\left(\frac{1-\operatorname{Cos} \beta_{m} t}{\beta_{m}}\right) \\
& +\frac{\operatorname{Cos} \alpha_{c} t-\operatorname{Cos} \beta_{m} t}{\beta_{m}^{2}-\alpha_{c}^{2}} \\
& -A_{k}\left(\frac{\beta_{m} \operatorname{Sin} \alpha_{c} t-\alpha_{c} \operatorname{Sin} \beta_{m} t}{\beta_{m}\left(\beta_{m}^{2}-\alpha_{c}^{2}\right)}\right) \\
& -B_{k}\left(\frac{\operatorname{Cosh} \alpha_{c} t-\operatorname{Cos} \beta_{m} t}{\alpha_{c}^{2}+\beta_{m}^{2}}\right) \\
& \left.-C_{k}\left(\frac{\beta_{m} \operatorname{Sinh} \alpha_{c} t-\alpha_{c} \operatorname{Sin} \beta_{m} t}{\beta_{m}\left(\alpha_{c}^{2}+\beta_{m}^{2}\right)}\right)\right\},
\end{aligned}
$$

where

$$
\alpha_{c}=\frac{\lambda_{k} c}{L}
$$

Thus using (27) in (12), one obtains

$$
\begin{aligned}
& V_{n}(x, t)=\sum_{m=1}^{\infty} P_{0}\left\{B_{f}(\lambda, k)\left(\frac{1-\operatorname{Cos} \beta_{m} t}{\beta_{m}}\right)\right. \\
&+ \frac{\operatorname{Cos} \alpha_{c} t-\operatorname{Cos} \beta_{m} t}{\beta_{m}^{2}-\alpha_{c}^{2}} \\
&-A_{k}\left(\frac{\beta_{m} \operatorname{Sin} \alpha_{c} t-\alpha_{c} \operatorname{Sin} \beta_{m} t}{\beta_{m}\left(\beta_{m}^{2}-\alpha_{c}^{2}\right)}\right) \\
&-B_{k}\left(\frac{\operatorname{Cosh} \alpha_{c} t-\operatorname{Cos} \beta_{m} t}{\alpha_{c}^{2}+\beta_{m}^{2}}\right) \\
&\left.-C_{k}\left(\frac{\beta_{m} \operatorname{Sinh} \alpha_{c} t-\alpha_{c} \operatorname{Sin} \beta_{m} t}{\beta_{m}\left(\alpha_{c}^{2}+\beta_{m}^{2}\right)}\right)\right\} \\
& \cdot\left\{\operatorname{Sin} \frac{\lambda_{m} x}{L}+A_{m} \operatorname{Cos} \frac{\lambda_{m} x}{L}\right. \\
&\left.+B_{m} \operatorname{Sinh} \frac{\lambda_{m} x}{L}+C_{m} \operatorname{Cosh} \frac{\lambda_{m} x}{L}\right\}
\end{aligned}
$$

Equation (29) represents the transverse displacement response to a distributed force moving at constant velocity of a nonuniform Rayleigh beam having arbitrary end support conditions.

4.2. Case II. If the inertia term is retained, then $\epsilon_{a} \neq 0$. This is termed the moving mass problem. In this case, the solution to the entire equation (22) is required. As an exact solution to this problem is impossible, a modification of Struble's technique discussed in [1] is employed. To this end, (22) is simplified and rearranged to take the form

$$
\begin{aligned}
& \ddot{W}_{m}(t)+\frac{\epsilon_{a} L R_{2}(m, k, t)}{1+\epsilon_{a} L R_{1}(m, k, t)} \dot{W}_{m}(t) \\
& +\frac{\left(\beta_{m}^{2}+\epsilon_{a} L R_{3}(m, k, t)\right)}{1+\epsilon_{a} L R_{1}(m, k, t)} W_{m}(t) \\
& =\left(\frac{\epsilon_{a} L^{2} g}{\lambda_{k} \Delta_{1}(m, k)}\right. \\
& \quad \times\left(B_{f}(\lambda, k)+\operatorname{Cos} \frac{\lambda_{k} c t}{L}\right. \\
& -A_{k} \operatorname{Sin} \frac{\lambda_{k} c t}{L}-B_{k} \operatorname{Cosh} \frac{\lambda_{k} c t}{L} \\
& \left.\left.-C_{k} \operatorname{Sinh} \frac{\lambda_{k} c t}{L}\right)\right) \times\left(1+\epsilon_{a} L R_{1}(m, k, t)\right)^{-1},
\end{aligned}
$$

where

$$
\begin{aligned}
R_{1}(m, k, t) & \\
= & Q_{1 a}(m, k)+\frac{1}{\pi} \sum_{n=0}^{\infty} \frac{\operatorname{Cos}(2 n+1) \pi c t}{2 n+1} Q_{1 b}(n, m, k) \\
& -\frac{1}{\pi} \sum_{n=0}^{\infty} \frac{\operatorname{Sin}(2 n+1) \pi c t}{2 n+1} Q_{1 \bar{c}}(n, m, k),
\end{aligned}
$$

$R_{2}(m, k, t)$

$$
\begin{gathered}
=2 c Q_{2 a}(m, k)+\frac{2 c}{\pi} \sum_{n=0}^{\infty} \frac{\operatorname{Cos}(2 n+1) \pi c t}{2 n+1} Q_{2 b}(n, m, k) \\
-\frac{2 c}{\pi} \sum_{n=0}^{\infty} \frac{\operatorname{Sin}(2 n+1) \pi c t}{2 n+1} Q_{2 \bar{c}}(n, m, k),
\end{gathered}
$$

$$
\begin{aligned}
& R_{3}(m, k, t) \\
&=c^{2} Q_{3 a}(m, k)+\frac{c^{2}}{\pi} \sum_{n=0}^{\infty} \frac{\operatorname{Cos}(2 n+1) \pi c t}{2 n+1} Q_{3 b}(n, m, k) \\
&-\frac{c^{2}}{\pi} \sum_{n=0}^{\infty} \frac{\operatorname{Sin}(2 n+1) \pi c t}{2 n+1} Q_{3 \bar{c}}(n, m, k), \\
& Q_{1 a}(m, k)=\frac{Q_{1 A}(m, k)}{\Delta_{1}(m, k)} \\
& Q_{2 a}(m, k)=\frac{Q_{2 A}(m, k)}{\Delta_{1}(m, k)} \\
& Q_{1 b}(n, m, k)=\frac{Q_{1 B}(n, m, k)}{\Delta_{1}(m, k)} \\
& Q_{2 b}(n, m, k)=\frac{Q_{2 B}(n, m, k)}{\Delta_{1}(m, k)}
\end{aligned}
$$




$$
\begin{aligned}
Q_{3 a}(m, k) & =\frac{Q_{3 A}(m, k)}{\Delta_{1}(m, k)}, \\
Q_{3 b}(n, m, k) & =\frac{Q_{3 B}(n, m, k)}{\Delta_{1}(m, k)}, \\
Q_{1 \bar{c}}(n, m, k) & =\frac{Q_{1 C}(n, m, k)}{\Delta_{1}(m, k)}, \\
Q_{2 \bar{c}}(n, m, k) & =\frac{Q_{2 C}(n, m, k)}{\Delta_{1}(m, k)}, \\
Q_{3 \bar{c}}(n, m, k) & =\frac{Q_{3 C}(n, m, k)}{\Delta_{1}(m, k)} .
\end{aligned}
$$

By means of this technique, one seeks the modified frequency corresponding to the frequency of the free system due to the presence of the distributed moving mass. An equivalent free system operator defined by the modified frequency then replaces (30). Thus, the right-hand side of (30) is set to zero, and a parameter $\epsilon^{*}<1$ is considered for any arbitrary mass ratio $\epsilon_{a}$, defined as

$$
\epsilon^{*}=\frac{\epsilon_{a}}{1+\epsilon_{a}} .
$$

Evidently,

$$
\epsilon_{a}=\epsilon^{*}+o\left(\epsilon^{*}\right)^{2},
$$

which implies

$$
\frac{1}{1+\epsilon^{*} L R_{1}(m, k, t)}=1-\epsilon^{*} L R_{1}(m, k, t),
$$

where

$$
\begin{aligned}
\mid \epsilon^{*} L\left(Q_{1 a}+\frac{1}{\pi} \sum_{n=0}^{\infty} \frac{\operatorname{Cos}(2 n+1) \pi c t}{2 n+1} Q_{1 b}\right. \\
\left.-\frac{1}{\pi} \sum_{n=0}^{\infty} \frac{\operatorname{Sin}(2 n+1) \pi c t}{2 n+1} Q_{1 \bar{c}}\right) \mid<1 .
\end{aligned}
$$

When we set $\epsilon^{*}=0$ a case corresponding to a situation when the inertia effect of the mass of the system is neglected is obtained and the solution of (30) can be written as

$$
W_{m}(t)=\psi_{m} \operatorname{Cos}\left(\beta_{m} t-\theta_{m}\right),
$$

where $\psi_{m}$ and $\theta_{m}$ are constants.

Since $\epsilon^{*}<1$, Struble's technique requires that the asymptotic solution of the homogeneous part of (30) be of the form given in [23] by Nayfey as

$$
\begin{aligned}
W_{m}(t)= & \psi(m, t) \operatorname{Cos}\left(\beta_{m} t-\theta(m, t)\right) \\
& +\epsilon^{*} W_{1}(m, t)+o\left(\eta^{2}\right),
\end{aligned}
$$

where $\psi(m, t)$ and $\theta(m, t)$ are slowly varying functions of time.
Substituting (36) and its derivatives into homogeneous part of (30) and taking into account (33), one obtains

$$
\begin{aligned}
& -2 \dot{\psi}(m, t) \beta_{m} \operatorname{Sin}\left(\beta_{m} t-\theta(m, t)\right) \\
& +2 \psi(m, t) \dot{\theta}(m, t) \beta_{m} \operatorname{Cos}\left(\beta_{m} t-\theta(m, t)\right) \\
& -2 c \epsilon^{*} L Q_{2 a}(m, k) \psi(m, t) \\
& \cdot \beta_{m} \operatorname{Sin}\left(\beta_{m} t-\theta(m, t)\right)-\frac{2 c \epsilon^{*} L \psi(m, t)}{\pi} \\
& \times \beta_{m} \sum_{n=0}^{\infty} \frac{\operatorname{Cos}(2 n+1) \pi c t}{2 n+1} \operatorname{Sin}\left(\beta_{m} t-\theta(m, t)\right) \\
& \times Q_{2 b}(n, m, k)+\frac{2 c \epsilon^{*} L \psi(m, t)}{\pi} \beta_{m} \\
& \times \sum_{n=0}^{\infty} \frac{\operatorname{Sin}(2 n+1) \pi c t}{2 n+1} Q_{2 \bar{c}}(n, m, k) \operatorname{Sin}\left(\beta_{m} t-\theta(m, t)\right) \\
& -\beta_{m}^{2} \epsilon^{*} L Q_{1 a}(m, k) \psi(m, t) \\
& \cdot \operatorname{Cos}\left(\beta_{m} t-\theta(m, t)\right)-\frac{\beta_{m}^{2} \epsilon^{*} L \psi(m, t)}{\pi} \\
& \times \sum_{n=0}^{\infty} \frac{\operatorname{Cos}(2 n+1) \pi c t}{2 n+1} Q_{1 b}(n, m, k) \operatorname{Cos}\left(\beta_{m} t-\theta(m, t)\right) \\
& +\frac{\epsilon^{*} L \psi(m, t)}{\pi} \sum_{n=0}^{\infty} \frac{\operatorname{Sin}(2 n+1) \pi c t}{2 n+1} \beta_{m}^{2} \operatorname{Cos}\left(\beta_{m} t-\theta(m, t)\right) \\
& \times Q_{1 \bar{c}}(n, m, k)+c^{2} \epsilon^{*} L Q_{3 a}(m, k) \psi(m, t) \\
& \times \operatorname{Cos}\left(\beta_{m} t-\theta(m, t)\right) \\
& +\frac{c^{2} \epsilon^{*} L \psi(m, t)}{\pi} \sum_{n=0}^{\infty} \frac{\operatorname{Cos}(2 n+1) \pi c t}{2 n+1} Q_{3 b}(n, m, k) \\
& \cdot \operatorname{Cos}\left(\beta_{m} t-\theta(m, t)\right)-\frac{c^{2} \epsilon^{*} L \psi(m, t)}{\pi} \\
& \times \sum_{n=0}^{\infty} \frac{\operatorname{Sin}(2 n+1) \pi c t}{2 n+1} \operatorname{Cos}\left(\beta_{m} t-\theta(m, t)\right) Q_{3 \bar{c}}(n, m, k)=0
\end{aligned}
$$

retaining terms to $o\left(\epsilon^{*}\right)$.

The variational equations are obtained by equating the coefficients of $\operatorname{Sin}\left(\beta_{m} t-\theta(m, t)\right)$ and $\operatorname{Cos}\left(\beta_{m} t-\theta(m, t)\right)$ terms on both sides of (37) to zero. Thus, noting the following trigonometric identities:

$$
\begin{aligned}
\frac{\operatorname{Cos}(2 n+1) \pi c t}{2 n+1} \operatorname{Sin}\left[\beta_{m} t-\theta(m, t)\right] \\
=\frac{1}{2} \operatorname{Sin}\left[\frac{(2 n+1) \pi c t}{2 n+1}+\beta_{m} t-\theta(m, t)\right] \\
\quad-\frac{1}{2} \operatorname{Sin}\left[\frac{(2 n+1) \pi c t}{2 n+1}-\beta_{m} t+\theta(m, t)\right]
\end{aligned}
$$




$$
\begin{aligned}
& \frac{\operatorname{Sin}(2 n+1) \pi c t}{2 n+1} \operatorname{Sin}\left[\beta_{m} t-\theta(m, t)\right] \\
& =\frac{1}{2} \operatorname{Cos}\left[\frac{(2 n+1) \pi c t}{2 n+1}-\beta_{m} t+\theta(m, t)\right] \\
& \quad-\frac{1}{2} \operatorname{Cos}\left[\frac{(2 n+1) \pi c t}{2 n+1}+\beta_{m} t-\theta(m, t)\right] \\
& \frac{\operatorname{Cos}(2 n+1) \pi c t}{2 n+1} \operatorname{Cos}\left[\beta_{m} t-\theta(m, t)\right] \\
& =\frac{1}{2} \operatorname{Cos}\left[\frac{(2 n+1) \pi c t}{2 n+1}+\beta_{m} t-\theta(m, t)\right] \\
& \quad+\frac{1}{2} \operatorname{Cos}\left[\frac{(2 n+1) \pi c t}{2 n+1}-\beta_{m} t+\theta(m, t)\right] \\
& \frac{\operatorname{Sin}(2 n+1) \pi c t}{2 n+1} \operatorname{Cos}\left[\beta_{m} t-\theta(m, t)\right] \\
& =\frac{1}{2} \operatorname{Sin}\left[\frac{(2 n+1) \pi c t}{2 n+1}+\beta_{m} t-\theta(m, t)\right] \\
& +\frac{1}{2} \operatorname{Sin}\left[\frac{(2 n+1) \pi c t}{2 n+1}-\beta_{m} t+\theta(m, t)\right],
\end{aligned}
$$

and neglecting terms that do not contribute to the variational equations, (37) reduces to

$$
\begin{aligned}
& -2 \dot{\psi}(m, t) \beta_{m} \operatorname{Sin}\left(\beta_{m} t-\theta(m, t)\right) \\
& +2 \psi(m, t) \dot{\theta}(m, t) \beta_{m} \operatorname{Cos}\left(\beta_{m} t-\theta(m, t)\right) \\
& -2 c \epsilon^{*} L Q_{2 a}(m, k) \psi(m, t) \beta_{m} \\
& \times \operatorname{Sin}\left(\beta_{m} t-\theta(m, t)\right)-\beta_{m}^{2} \epsilon^{*} L Q_{1 a}(m, k) \psi(m, t) \\
& \times \operatorname{Cos}\left(\beta_{m} t-\theta(m, t)\right) \\
& +c^{2} \epsilon^{*} L Q_{3 a}(m, k) \psi(m, t) \operatorname{Cos}\left(\beta_{m} t-\theta(m, t)\right) \\
& =0 .
\end{aligned}
$$

Then, the variational equations are, respectively,

$$
\begin{aligned}
& -2 \dot{\psi}(m, t) \beta_{m}-2 c \epsilon^{*} L Q_{2 a}(m, k) \psi(m, t) \beta_{m}=0, \\
& 2 \psi(m, t) \dot{\theta}(m, t) \beta_{m}-\beta_{m}^{2} \epsilon^{*} L Q_{1 a}(m, k) \psi(m, t) \\
& \quad+c^{2} \epsilon^{*} L Q_{3 a}(m, k) \psi(m, t)=0 .
\end{aligned}
$$

Solving (39) and (40), respectively, one obtains

$$
\psi(m, t)=\psi_{m} e^{-c \epsilon^{*} L Q_{2 a}(m, k) t},
$$

$\theta(m, t)$

$$
=\frac{\beta_{m} \epsilon^{*} L Q_{1 a}(m, k)}{2} t-\frac{c^{2} \epsilon^{*} L Q_{3 a}(m, k)}{2 \beta_{m}} t+\theta_{m},
$$

where $\psi_{m}$ and $\theta_{m}$ are constants.

Therefore, when the effects of the mass of the particle are considered, the first approximation to the homogeneous system is given as

$$
W_{m}(t)=\psi(m, t) \operatorname{Cos}\left(\omega_{m} t-\theta_{m}\right)
$$

where

$$
\omega_{m}=\frac{2 \beta_{m}^{2}-\epsilon^{*} L\left\{\beta_{m}^{2} Q_{1 a}(m, k)-c^{2} Q_{3 a}(m, k)\right\}}{2 \beta_{m}}
$$

is called the modified natural frequency representing the frequency of the free system due to the presence of the moving mass.

Thus, to solve the nonhomogeneous equation (30), the differential operator which acts on $W_{m}(t)$ is replaced by the equivalent free system operator defined by the modified frequency $\omega_{m}$, that is,

$$
\begin{gathered}
\ddot{W}_{m}(t)+\omega_{m}^{2} W_{m}(t) \\
=P_{1}\left[B_{f}(\lambda, k)+\operatorname{Cos} \alpha_{c} t-A_{k} \operatorname{Sin} \alpha_{c} t\right. \\
\left.\quad-B_{k} \operatorname{Cosh} \alpha_{c} t-C_{k} \operatorname{Sinh} \alpha_{c} t\right],
\end{gathered}
$$

where

$$
P_{1}=\frac{\epsilon^{*} L^{2} g}{\lambda_{k} \Delta_{1}(m, k)}
$$

Solving (48) in conjunction with the initial condition, one obtains expression for $W_{m}(t)$. Thus, in view of (12)

$$
\begin{gathered}
V_{n}(x, t) \quad \sum_{m=1}^{\infty} P_{1}\left\{B_{f}(\lambda, k)\left(\frac{1-\operatorname{Cos} \omega_{m} t}{\omega_{m}}\right)\right. \\
+\frac{\operatorname{Cos} \alpha_{c} t-\operatorname{Cos} \omega_{m} t}{\omega_{m}^{2}-\alpha_{c}^{2}} \\
-A_{k}\left(\frac{\omega_{m} \operatorname{Sin} \alpha_{c} t-\alpha_{c} \operatorname{Sin} \omega_{m} t}{\omega_{m}\left(\omega_{m}^{2}-\alpha_{c}^{2}\right)}\right) \\
-B_{k}\left(\frac{\operatorname{Cosh} \alpha_{c} t-\operatorname{Cos} \omega_{m} t}{\alpha_{c}^{2}+\omega_{m}^{2}}\right) \\
\left.-C_{k}\left(\frac{\omega_{m} \operatorname{Sinh} \alpha_{c} t-\alpha_{c} \operatorname{Sin} \omega_{m} t}{\omega_{m}\left(\alpha_{c}^{2}+\omega_{m}^{2}\right)}\right)\right\} \\
+\left\{\operatorname{Sin} \frac{\lambda_{m} x}{L}+A_{m} \operatorname{Cos} \frac{\lambda_{m} x}{L}\right. \\
\left.+B_{m} \operatorname{Sinh} \frac{\lambda_{m} x}{L}+C_{m} \operatorname{Cosh} \frac{\lambda_{m} x}{L}\right\}
\end{gathered}
$$

which represents the transverse displacement response to a moving distributed mass of a nonuniform Rayleigh beam having arbitrary end support conditions.

\section{Illustrative Examples}

In this section, practical examples of classical boundary conditions are used to illustrate the analyses presented in this paper. 
5.1. Clamped-Clamped Nonuniform Rayleigh Beam. The boundary conditions of a nonuniform Rayleigh beam clamped at both ends $x=0$ and $x=L$ are given by

$$
\begin{gathered}
V_{n}(0, t)=0=V_{n}(L, t), \\
\frac{\partial V_{n}(0, t)}{\partial x}=0=\frac{\partial V_{n}(L, t)}{\partial x}
\end{gathered}
$$

and hence for normal modes

$$
\begin{gathered}
U_{m}(0)=0=U_{m}(L), \\
\frac{\partial U_{m}(0)}{\partial x}=0=\frac{\partial U_{m}(L)}{\partial x}
\end{gathered}
$$

which implies

$$
\begin{gathered}
U_{k}(0)=0=U_{k}(L), \\
\frac{\partial U_{k}(0)}{\partial x}=0=\frac{\partial U_{k}(L)}{\partial x} .
\end{gathered}
$$

Applications of (52) to (20) yield

$$
\begin{aligned}
A_{m}=\frac{\operatorname{Sinh} \lambda_{m}-\operatorname{Sin} \lambda_{m}}{\operatorname{Cos} \lambda_{m}-\operatorname{Cosh} \lambda_{m}} & =\frac{\operatorname{Cos} \lambda_{m}-\operatorname{Cosh} \lambda_{m}}{\operatorname{Sin} \lambda_{m}-\operatorname{Sinh} \lambda_{m}}=-C_{m}, \\
B_{m} & =-1 .
\end{aligned}
$$

The frequency equation becomes

$$
\operatorname{Cos} \lambda_{m} \operatorname{Cosh} \lambda_{m}=1
$$

It follows that expression for $A_{k}, B_{k}, C_{k}$ and the corresponding frequency equations are obtained by a simple interchange of $m$ with $k$ in (54) and (55).

Substituting (54) and (55) into (29) and (50), one obtains the displacement response, respectively, to a moving distributed force and a moving distributed mass of a clampedclamped nonuniform Rayleigh beam.

\subsection{One End Clamped and One End Free Condition-Cantilever} Beam. As a second example, at end $x=0$, the beam is taken to be clamped and, at the end $x=L$, the beam is free. Thus, the boundary conditions can be written as

$$
\begin{gathered}
V_{n}(0, t)=0=\frac{\partial}{\partial x} V_{n}(0, t), \\
\frac{\partial^{2}}{\partial x^{2}} V_{n}(L, t)=0=\frac{\partial^{3}}{\partial x^{3}} V_{n}(L, t) .
\end{gathered}
$$

And for normal modes

$$
\begin{gathered}
U_{m}(0)=0=\frac{d}{d x} U_{m}(0), \\
\frac{d^{2}}{d x^{2}} U_{m}(L)=0=\frac{d^{3}}{d x^{3}} U_{m}(L)
\end{gathered}
$$

which implies that

$$
\begin{gathered}
U_{k}(0)=0=\frac{d}{d x} U_{k}(0), \\
\frac{d^{2}}{d x^{2}} U_{k}(L)=0=\frac{d^{3}}{d x^{3}} U_{k}(L) .
\end{gathered}
$$

Application of (58) to (21) yields

$$
\begin{aligned}
A_{k} & =-\frac{\operatorname{Sin} \lambda_{k}+\operatorname{Sinh} \lambda_{k}}{\operatorname{Cos} \lambda_{k}+\operatorname{Cosh} \lambda_{k}}=\frac{\operatorname{Cos} \lambda_{k}+\operatorname{Cosh} \lambda_{k}}{\operatorname{Sin} \lambda_{k}-\operatorname{Sinh} \lambda_{k}} \\
& =-C_{k}, \\
B_{k} & =-1 .
\end{aligned}
$$

And the frequency equation for both end conditions is

$$
\operatorname{Cos} \lambda_{k} \operatorname{Cosh} \lambda_{k}=-1 \text {. }
$$

It follows that expression for $A_{m}, B_{m}, C_{m}$ and the corresponding frequency equations are obtained by a simple interchange of $k$ with $m$ in (59) and (60).

Substituting (59) and (60) into (29) and (50), one obtains the displacement response, respectively, to a moving distributed force and a moving distributed mass of a clampedfree nonuniform Rayleigh beam.

\section{Discussion of Closed Form Solutions}

The response amplitude of a dynamical system such as this may grow without bound. Conditions under which this happens are termed resonance conditions. For both illustrative examples, we observe that the nonuniform Rayleigh beam traversed by a moving distributed force at constant velocity reaches a state of resonance whenever

$$
\beta_{m}=\alpha_{c}
$$

while the same beam under the action of a moving distributed mass experiences resonance effect whenever

$$
\omega_{m}=\alpha_{c}
$$

Evidently,

$$
\omega_{m}=\beta_{m}\left[1-\frac{\epsilon^{*} L}{2}\left\{Q_{1 a}(m, k)-\frac{c^{2} Q_{3 a}(m, k)}{\beta_{m}^{2}}\right\}\right]=\alpha_{c} .
$$

Equations (61) and (63) show that for the same natural frequency, the critical speed for the same system consisting of a nonuniform Rayleigh beam resting on an elastic foundation and traversed by a moving distributed force is greater than that traversed by a moving distributed mass. Thus resonance is reached earlier in the moving distributed mass system than in the moving distributed force system. 


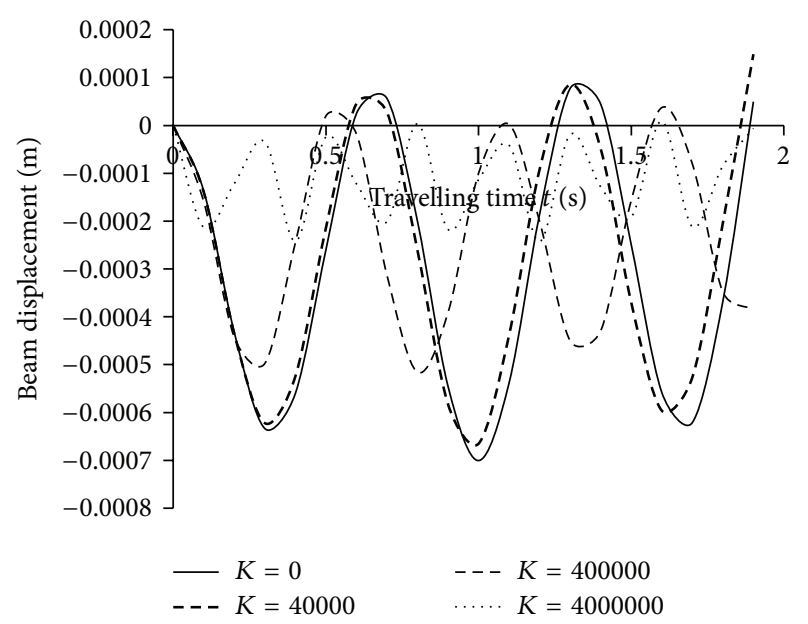

Figure 1: Displacement response of a clamped-clamped nonuniform Rayleigh beam under distributed forces for various values of foundation moduli $K$.

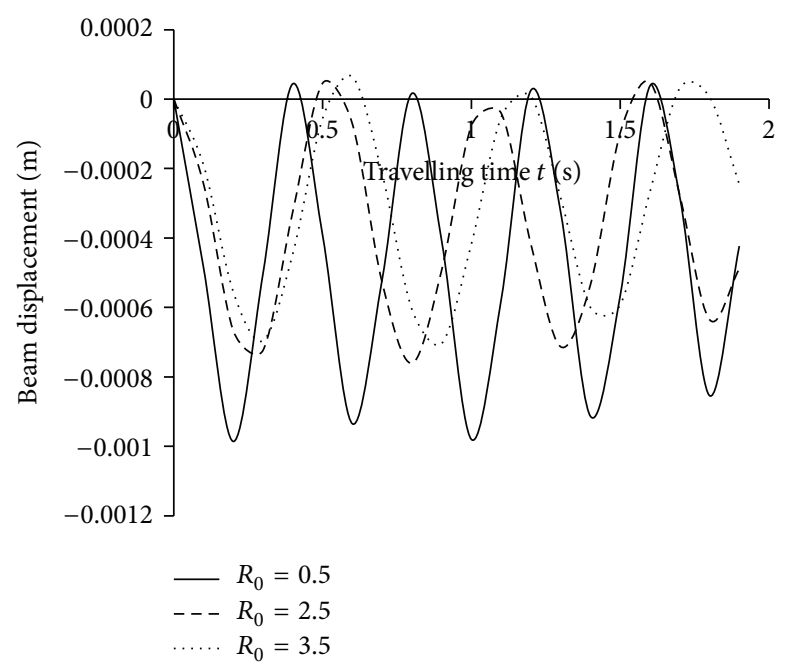

FIGURE 2: Displacement response of a clamped-clamped nonuniform Rayleigh beam under distributed forces for various values of rotatory inertia correction factor $R_{0}$.

\section{Numerical Results and Discussion}

In order to illustrate the foregoing analysis, the nonuniform Rayleigh beam of length $L=12.192 \mathrm{~m}$ is considered. The load velocity $c=8.128 \mathrm{~ms}^{-1}, E=2109 \times 10^{9} \mathrm{~kg} / \mathrm{m}$, and mass ratio $\epsilon_{a}=0.2$. The values of the rotatory inertia correction factor $R_{0}$ are varied between 0.5 and 9.5 , while the values of the foundation moduli $K$ are varied between 0 and $4000000 \mathrm{Nm}^{2}$. The displacement response of the nonuniform Rayleigh beam is calculated and graphs are plotted for beam response against time for values of rotatory inertia correction factor $R_{0}$ and foundation moduli constant $K$.

The transverse displacement response of the nonuniform clamped-clamped Rayleigh beam to distributed forces for various values of foundation moduli $K$ and fixed value of rotatory inertia correction factor $R_{0}=5$ is displayed

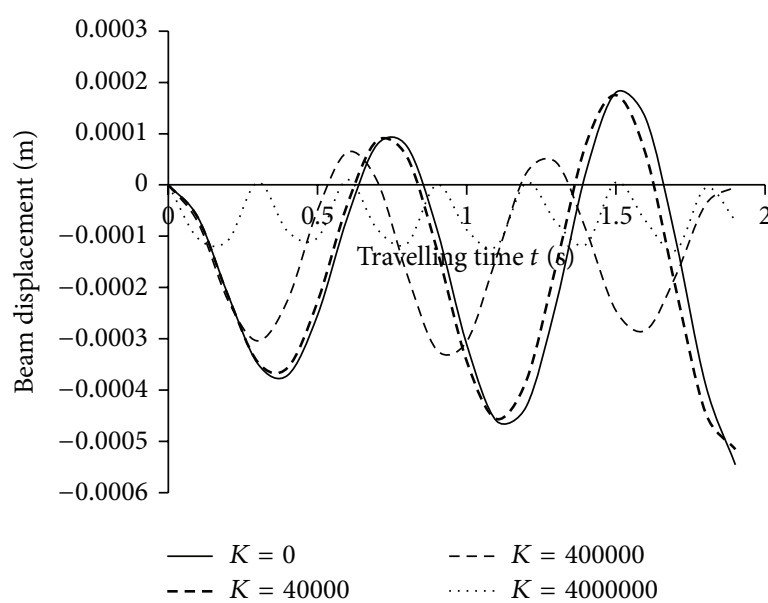

FIGURE 3: Displacement response of a clamped-clamped nonuniform Rayleigh beam to distributed masses for various values of foundation moduli $K$.

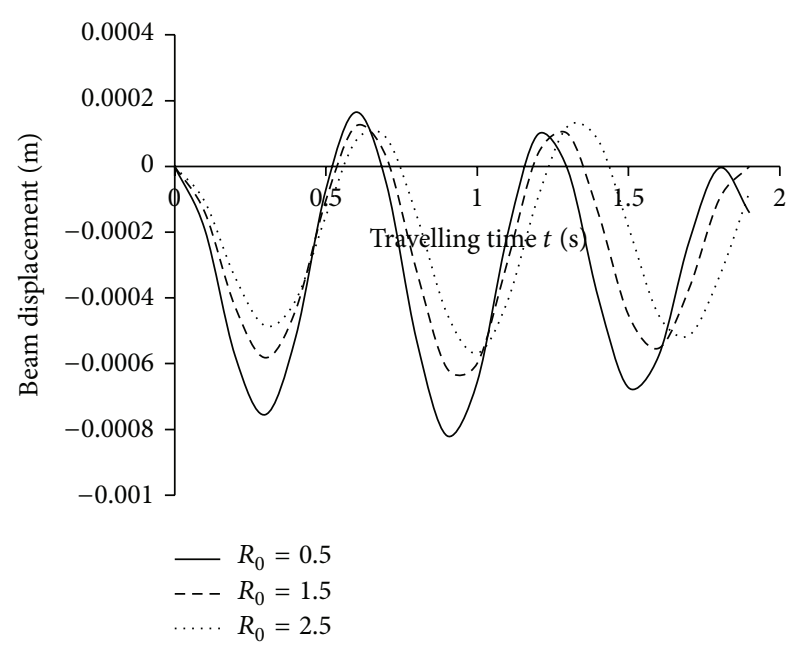

FIGURE 4: Displacement response of a clamped-clamped nonuniform Rayleigh beam under distributed masses for various values of rotatory inertia correction factor $R_{0}$.

in Figure 1. It is seen from the figure that as the values of the foundation moduli increase, the response amplitude of the nonuniform clamped-clamped Rayleigh beam under the action of distributed forces decreases. The same result is obtained when the same nonuniform clamped-clamped Rayleigh beam is traversed by moving distributed masses as shown in Figure 3. In Figure 2, the response of the clampedclamped nonuniform Rayleigh beam to distributed forces for various values of rotatory inertia correction factor $R_{0}$ and fixed value of foundation moduli $K=40000$ is depicted. It is seen that the deflection of the beam decreases with the increase in the rotatory inertia correction factor. The same behaviour characterizes the deflection profile of the nonuniform clamped-clamped Rayleigh beam when it is traversed by moving distributed masses as shown in Figure 4 . Figure 5 depicts the comparison of the transverse displacement response for moving distributed force and moving 


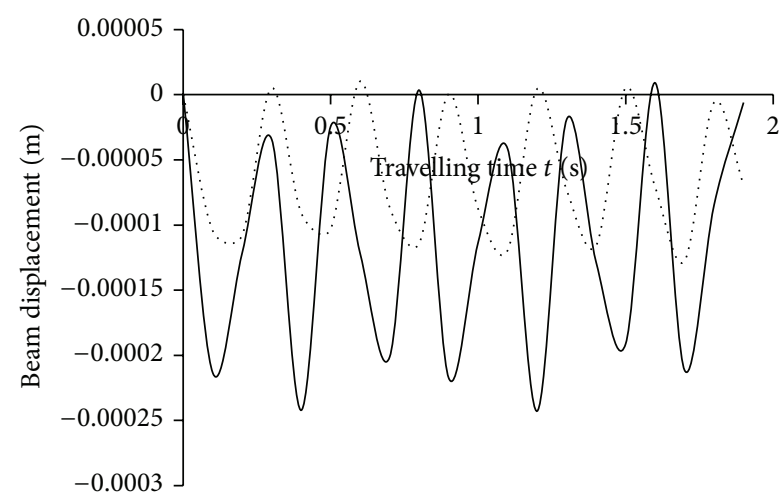

- Moving force
$\ldots .$.

Figure 5: Comparison of the deflection of moving distributed force and moving distributed mass for nonuniform clamped-clamped Rayleigh beam, $K=4000000, R_{0}=5$.

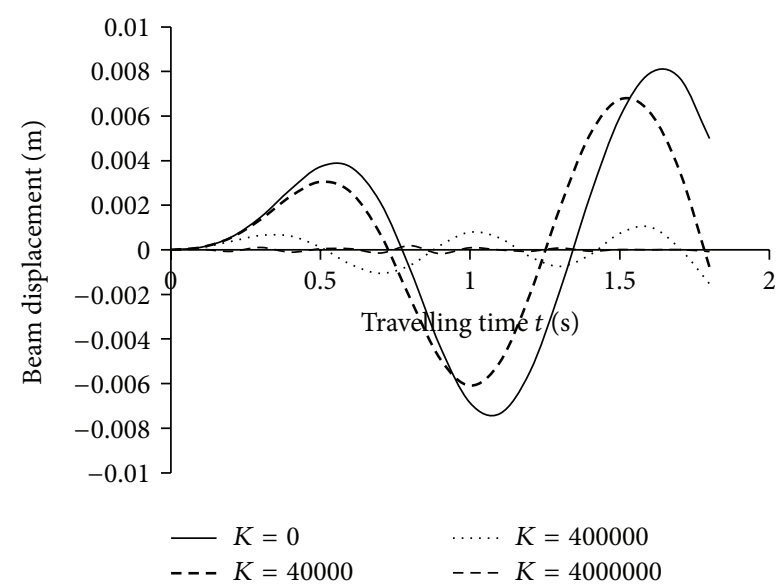

FIGURE 6: Displacement response of a nonuniform clamped-free Rayleigh beam under distributed forces for various values of foundation moduli $K$.

distributed mass cases of the nonuniform clamped-clamped Rayleigh beam for fixed values of foundation moduli $K=$ 4000000 and rotatory inertia correction factor $R_{0}=5$. It is observed that the response amplitude of the moving distributed force is greater than that of the moving distributed mass problem.

For the second illustrative example, the deflection profile of the nonuniform clamped-free Rayleigh beam to moving distributed forces for various values of foundation moduli $K$ and fixed value of rotatory inertia correction factor $R_{0}=5$ is displayed in Figure 6. It is seen that as the values of the foundation moduli increase, the deflection of the nonuniform clamped-free beam under the action of distributed forces decreases. The same results are obtained when the same nonuniform clamped-free Rayleigh beam is traversed by moving distributed masses as shown in Figure 8 . In Figure 7, the response of the nonuniform clamped-free

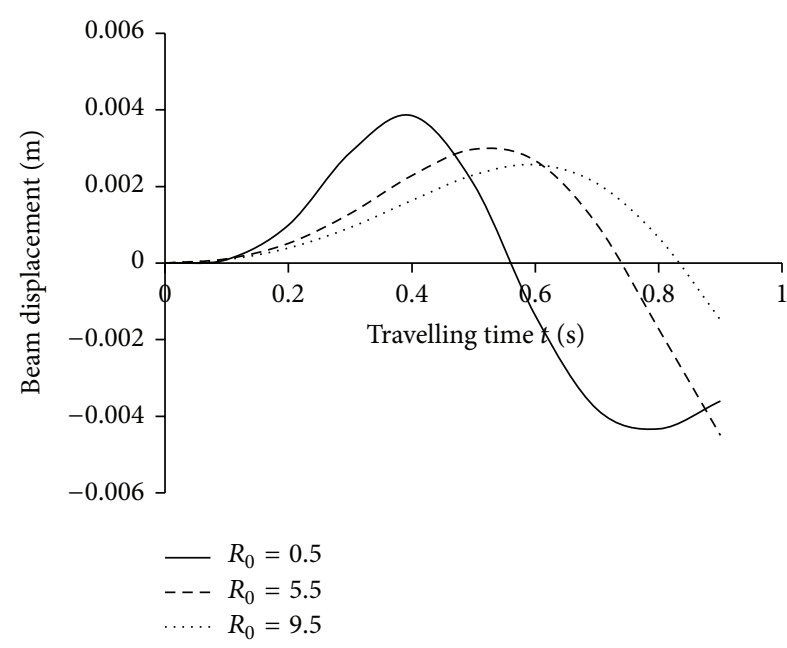

Figure 7: Displacement response of a nonuniform clamped-free Rayleigh beam under distributed forces for various values of rotatory inertia correction factor $R_{0}$.

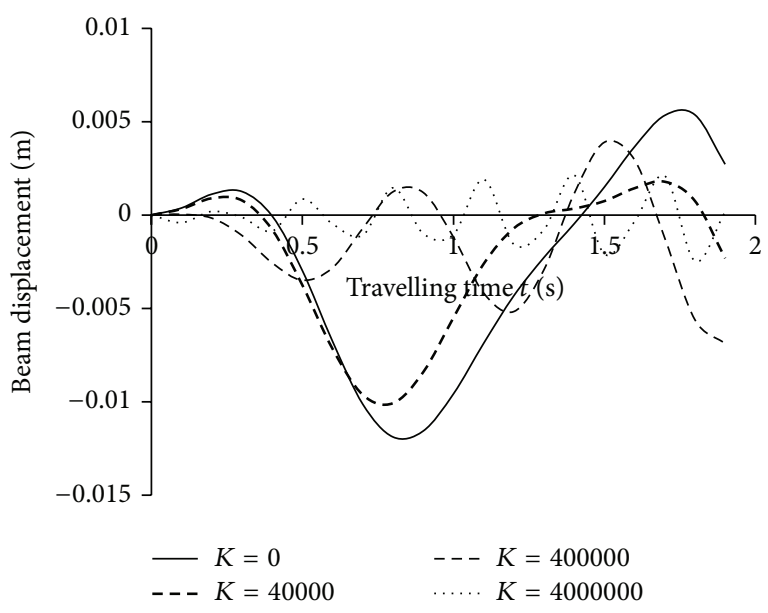

FIGURE 8: Displacement response of a nonuniform clamped-free Rayleigh beam under distributed masses for various values of foundation moduli $K$.

Rayleigh beam to moving distributed forces for various values of rotatory inertia correction factor $R_{0}$ and fixed value of foundation moduli $K=40000$ is depicted. It is observed that the deflection of the beam decreases with the increase in the rotatory inertia correction factor. The same behaviour characterizes the deflection profile of the same nonuniform clamped-free Rayleigh beam when it is traversed by moving distributed mass as displayed in Figure 9. Figure 10 depicts the comparison of the transverse displacement response for moving distributed force and moving distributed mass cases of the nonuniform clamped-free Rayleigh beam for fixed values of foundation moduli $K=4000000$ and rotatory inertia correction factor $R_{0}=5$. Clearly, the response amplitude of the moving distributed mass is greater than that of the moving distributed force problem. 


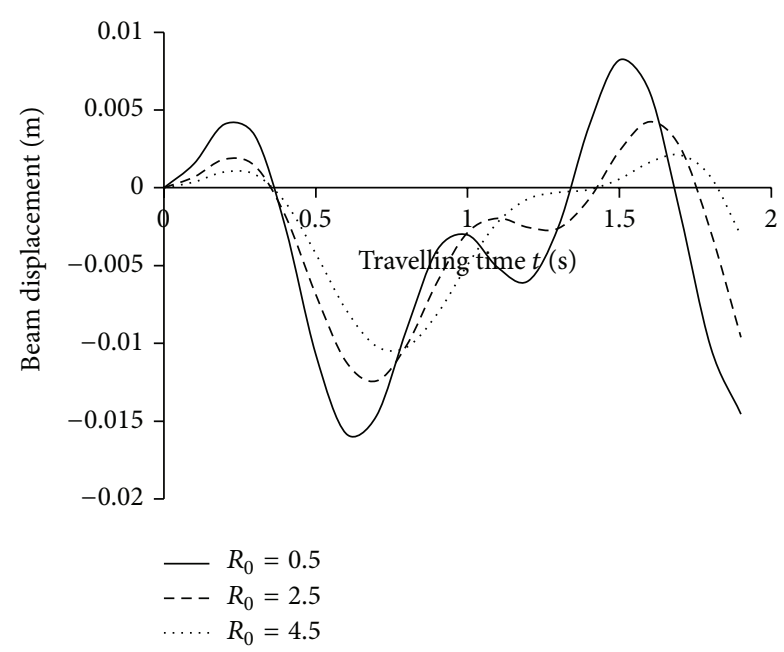

FIGURE 9: Deflection profile of a clamped-free nonuniform Rayleigh beam under distributed masses for various values of rotatory inertia correction factor $R_{0}$.

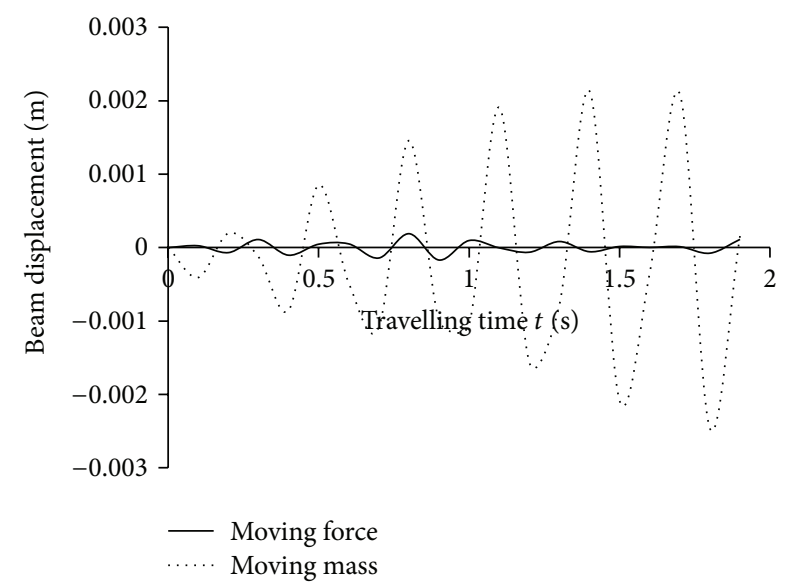

FIGURE 10: Comparison of the deflection of moving distributed force and moving distributed mass cases of a nonuniform clamped-free Rayleigh beam, $K=4000000, R_{0}=5$.

\section{Conclusion}

This paper investigated the flexural vibration of a finite nonuniform Rayleigh beam under travelling distributed loads. Both gravity and inertia effects of the distributed loads are taken into consideration. The versatile technique due to Galerkin suitable for all variants of classical boundary conditions was employed to reduce the governing fourthorder partial differential equation with variable coefficients to a sequence of second-order ordinary differential equations. These series of equations were treated using a modification of the asymptotic method of Struble and integral transformations. It is shown that an increase in the values of foundation moduli and rotatory inertia correction factor reduces the response amplitudes of both the clampedclamped nonuniform Rayleigh beam and the clamped-free nonuniform Rayleigh beam.
Analysis of the closed form solutions for both clampedclamped and clamped-free boundary conditions showed that the critical speed for the moving distributed mass problem is smaller than that for the moving distributed force problem. Hence resonance is reached earlier in the former. Furthermore, for fixed $R_{0}$ and $K$, the transverse deflections of the nonuniform Rayleigh beam with clamped-free end conditions which is under the action of moving distributed masses are higher than when the same elastic system is under the action of moving distributed forces, while for the same system with clamped-clamped end conditions, the deflection under the action of moving distributed forces is higher than when the system is under the action of moving distributed masses. Showing that the moving force solution is not always an upper bound to the moving mass problem, therefore, the inertia effect of the moving load must always be taken into consideration.

\section{Conflict of Interests}

The authors declare that there is no conflict of interests regarding the publication of this paper.

\section{References}

[1] S. T. Oni and E. Ayankop-Andi, "Response of a simply supported non-uniform Rayleigh beam to travelling distributed loads," Journal of the Nigerian Mathematical Society. In press.

[2] S. Sadiku and H. H. E. Leipholz, "On the dynamics of elastic systems with moving concentrated masses," Ingenieur-Archiv, vol. 57 , no. 3, pp. 223-242, 1987.

[3] S. T. Oni, "Flexural vibrations under moving loads of Isotropic rectangular plates on a non-Winkler elastic foundation," Journal of the Nigerian Society of Engineers, vol. 35, no. 1, pp. 18-27, 2000.

[4] J. A. Gbadeyan and S. T. Oni, "Dynamic behaviour of beams and rectangular plates under moving loads," Journal of Sound and Vibration, vol. 182, no. 5, pp. 677-695, 1995.

[5] M. H. Huang and D. P. Thambiratnam, "Deflection response of plate on Winkler foundation to moving accelerated loads," Engineering Structures, vol. 23, no. 9, pp. 1134-1141, 2001.

[6] H. P. Lee and T. Y. Ng, "Transverse vibration of a plate moving over multiple point supports," Applied Acoustics, vol. 47, no. 4, pp. 291-301, 1996.

[7] G. G. Adams, "Critical speeds and the response of a tensioned beam on an elastic foundation to repetitive moving loads," International Journal of Mechanical Sciences, vol. 37, no. 7, pp. 773-781, 1995.

[8] Y. H. Chen and C. Y. Li, "Dynamic response of elevated highspeed railway," Journal of Bridge Engineering, vol. 5, no. 2, pp. 124-130, 2000.

[9] E. Savin, "Dynamic amplification factor and response spectrum for the evaluation of vibrations of beams under successive moving loads," Journal of Sound and Vibration, vol. 248, no. 2, pp. 267-288, 2001.

[10] G. V. Rao, "Linear dynamics of an elastic beam under moving loads," Journal of Vibration and Acoustics, vol. 122, no. 3, pp. 281289, 2000.

[11] M. R. Shadnam, F. R. Rofooei, M. Mofid, and B. Mehri, "Periodicity in the response of nonlinear plate, under moving mass," Thin-Walled Structures, vol. 40, no. 3, pp. 283-295, 2002. 
[12] S. T. Oni and T. O. Awodola, "Dynamic behaviour under moving Concentrated masses of elastically supported finite Bernoulli-Euler beam on Winkler foundation," Journal of the Nigerian Mathematical Society, vol. 28, pp. 49-76, 2009.

[13] S. T. Oni, "Response of a non-uniform beam resting on an elastic foundation to several masses," Abacus, vol. 24, no. 2, 1996.

[14] S. T. Oni and T. O. Awodola, "Vibrations under a moving load of a non-uniform Rayleigh beam on variable elastic foundation," Journal of the Nigerian Association of Mathematical Physics, vol. 7, pp. 191-206, 2003.

[15] S. T. Oni and B. Omolofe, "Dynamic behaviour of nonuniform Bernoulli-Euler beams subjected to concentrated loads travelling at varying velocities," Abacus A, vol. 32, no. 2, pp. 165191, 2005.

[16] E. J. Sapountzakis and G. C. Tsiatas, "Elastic flexural buckling analysis of composite beams of variable cross-section by BEM," Engineering Structures, vol. 29, no. 5, pp. 675-681, 2007.

[17] A. Jimoh, Dynamic response to moving concentrated loads of Bernoulli-Euler beams resting on Bi-parametric subgrades [M.S. thesis], Federal University of Technology, Akure, Nigeria, 2013.

[18] E. Esmailzadeh and M. Ghorashi, "Vibration analysis of beams traversed by uniform partially distributed moving masses," Journal of Sound and Vibration, vol. 184, no. 1, pp. 9-17, 1995.

[19] J. A. Gbadeyan and M. S. Dada, "Dynamic response of a Mindlin elastic rectangular plate under a distributed moving mass," International Journal of Mechanical Sciences, vol. 48, no. 3, pp. 323-340, 2006.

[20] R. Bogacz and W. Czyczula, "Response of beam on visco-elastic foundation to moving distributed load," Journal of Theoretical and Applied Mechanics, vol. 46, no. 4, pp. 763-775, 2008.

[21] L. Fryba, Vibrations of Solids and Structures under Moving Loads, Noordhoff, Groningen, The Netherlands, 1972.

[22] S. W. Williams, Theory and Problems of Mechanical Vibrations, McGraw-Hill, New York, NY, USA, 1964.

[23] A. H. Nayfey, Perturbation Methods, John Wiley \& Sons, New York, NY, USA, 1973. 


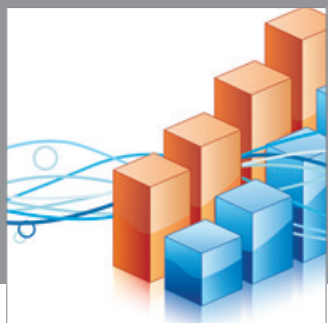

Advances in

Operations Research

mansans

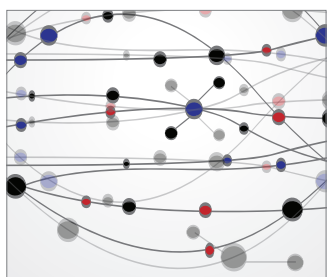

The Scientific World Journal
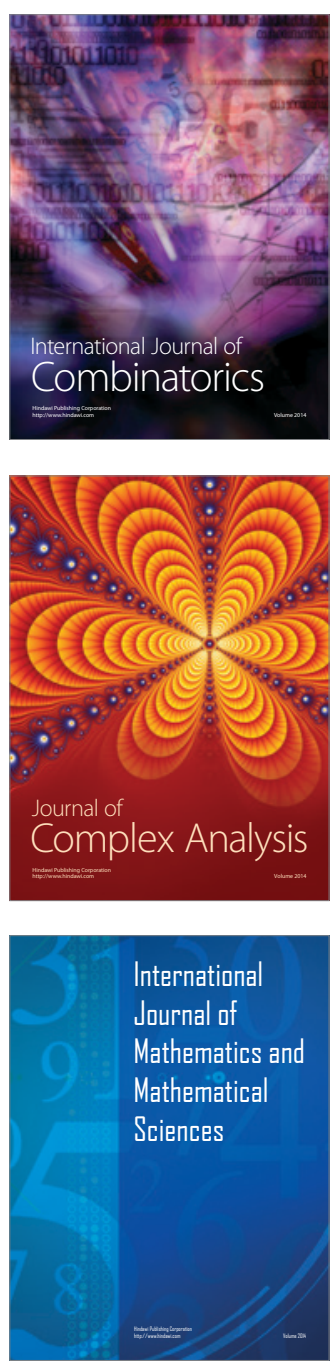
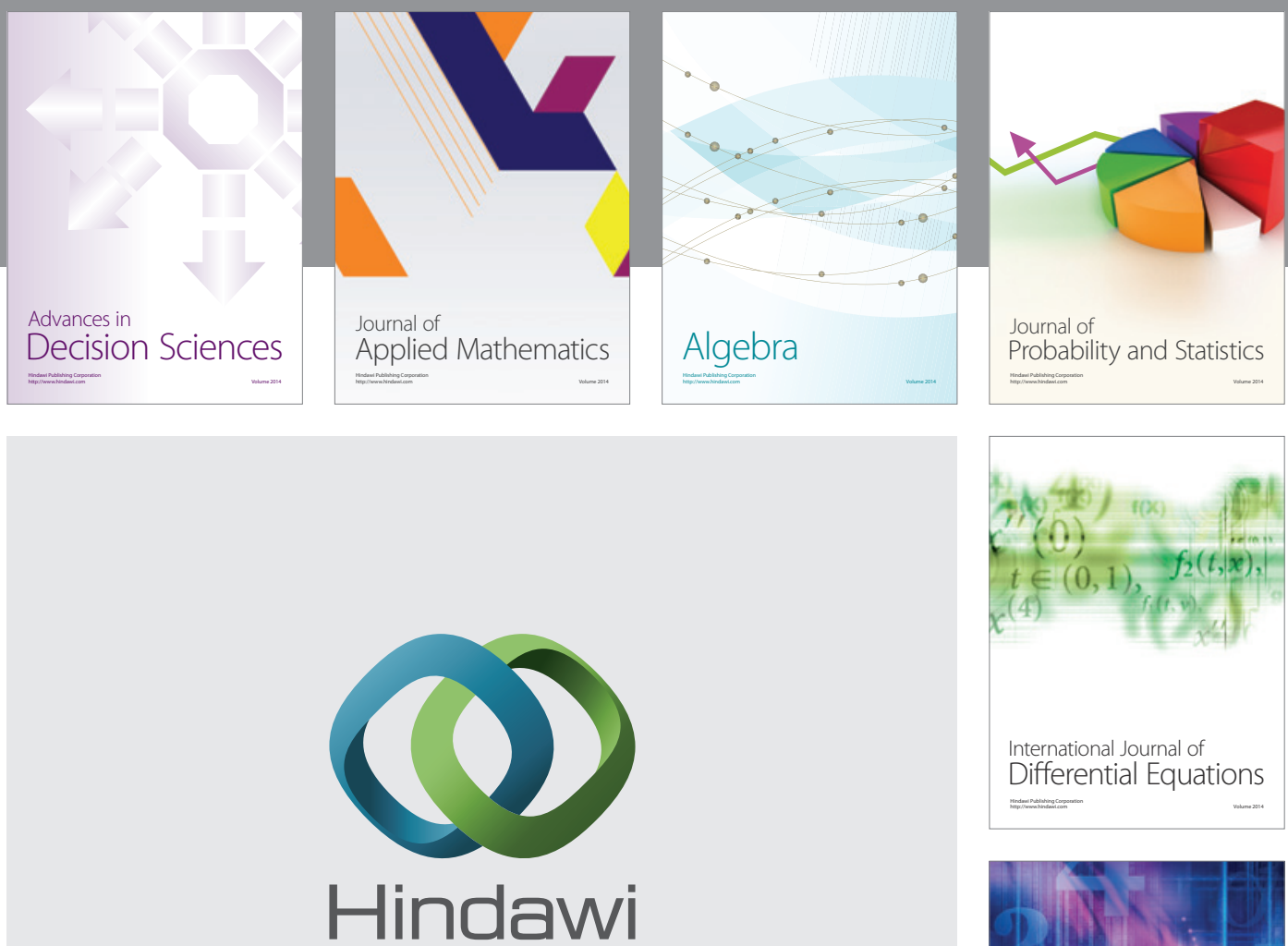

Submit your manuscripts at http://www.hindawi.com
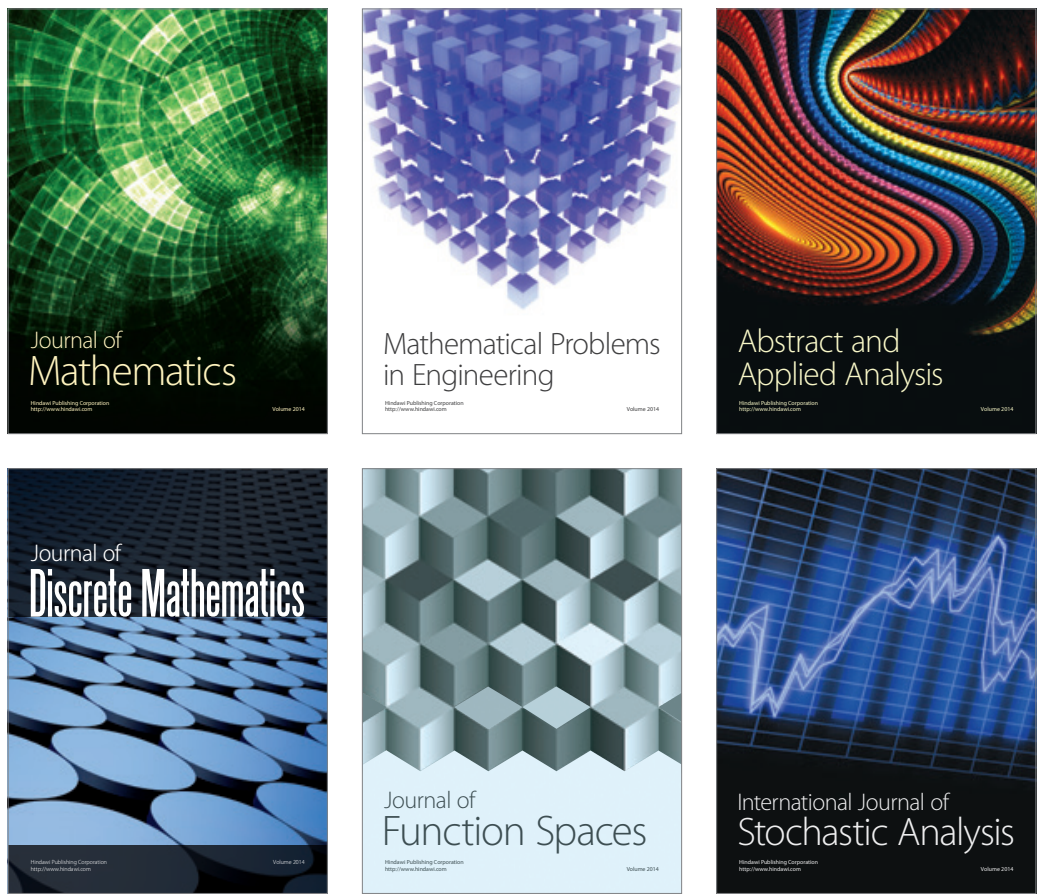

Journal of

Function Spaces

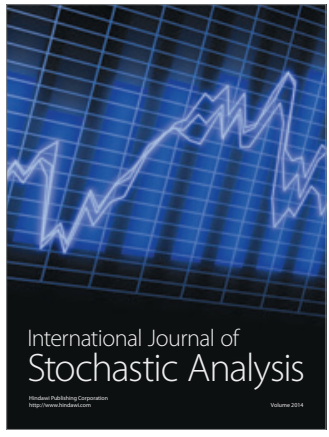

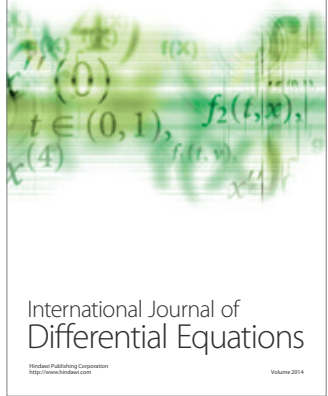
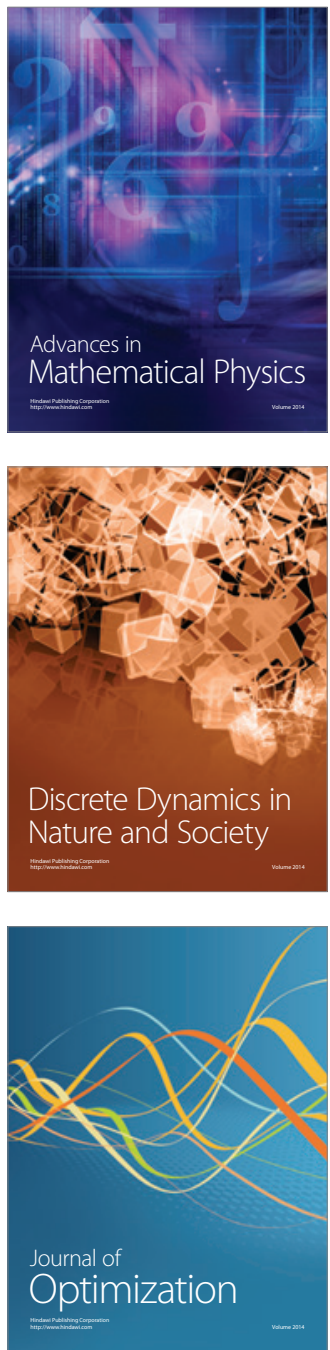